\title{
A CoMP soft handover scheme for LTE systems in high speed railway
}

Wantuan Luo ${ }^{1,2^{*}}$, Ruiqiang Zhang ${ }^{1}$ and Xuming Fang ${ }^{1}$

\begin{abstract}
With the development of high-speed railway and public growing demand on data traffic, people pay much more attention to provide high data rate and high reliable services under high mobility circumstance. Due to the higher data rate and lower system latency, long-term evolution (LTE) has been chosen as the next generation's evolution of railway mobile communication system by the International Union of Railways. However, there are still many problems to be solved in the high mobility applications of LTE, especially the higher handover failure probability, which seriously degrades the reliability of railway communication. This article proposes an optimized handover scheme, in which the coordinated multiple point transmission technology and dual vehicle station coordination mechanism are applied to improve the traditional hard handover performance of LTE. The scheme enables the high speed train to receive signals from both adjacent base stations and obtain diversity gain when it moves through the overlapping areas, so it improves the quality of the received signal and provides reliable communication between train and ground eNodeBs. Numerical analysis and simulation results show that the proposed scheme can decrease the outage probability remarkably during handover and guarantee the reliability of train to ground communication.
\end{abstract}

Keywords: LTE, soft handover, coordinate multiple point transmission (CoMP), high mobility, outage probability

\section{Introduction}

Due to the lower energy consumption, less environmental pollution, larger transport capacity and more safety, railway transportation plays an important role for the development of country. Japanese Shinkansen, French TGV, German ICE and China Railway have achieved remarkable successes. Nowadays, the development of high-speed railway makes people's lives more and more convenient. Meanwhile, it puts forward higher requirements on high-2 speed railway communication services. The existing GSM for Railway (GSM-R) network is mainly based on the second-generation Global System for Mobile Communications (GSM), and its data rate is too low to meet the broadband mobile communication access and other value-added service demands of passengers. In order to provide broadband services and applications for users not only at home but also on trip, long-term evolution (LTE) has been chosen as the next

\footnotetext{
* Correspondence: Wantuan.luo@gmail.com

${ }^{1}$ Institute of Mobile Communications, Southwest Jiaotong University, Chengdu, 610031, China

Full list of author information is available at the end of the article
}

generation's evolution of railway mobile communication system by International Union of Railways (UIC), which supports significant higher data rates and lower system latency. This article focuses on the applications of LTE technology in railway broadband wireless communication network.

Normally, the main problems caused by user's highspeed movement in cellular wireless communication system are over-frequent handover, Doppler shift and large penetration loss, among which over-frequent handover needs to be paid special attention as it seriously affects the communication quality of service $(\mathrm{QoS})$ and traffic reliability. Currently only traditional hard handover scheme is supported in LTE, which encounters two challenges under high speed movement circumstance. On the one hand, the handover delay caused by hard handover is relatively large. The high-speed train passes through the overlapping areas so fast that the handover procedure can not be accomplished timely. On the other hand, the speed of MRS is so fast that it would miss the optimal handover position, which degrades the handover success probability. In order to overcome the 
challenges mentioned above, the existing handover scheme of LTE should be optimized to improve the handover success probability in high-speed movement circumstance.

Currently, more and more researches focus on the broadband communication access issues in railway communication. In [1], a fast handover algorithm suitable for dedicated passenger line is devised by setting a new neighboring list. However, the optimization of system parameters has limited improvement on the overall performance of system. A novel moving extended cell (MEC) concept is introduced in [2,3], which is based on user centric virtual groups of adjacent cells that transmit the same data to the user. The MEC concept utilizes a mechanism for restructuring the virtual multi-cell area according to the user's mobility pattern. This handover scheme is supposed to support high end-user mobility in a $60 \mathrm{GHz}$ broadband pico-cellular radio-over-fiber network. Jeon and Sanghoon [4], Pabst et al. [5] have introduced relays to the process of handover in cellular networks in order to improve handover performance. In [6,7], multiple radios in devices are exploited to eliminate handover latency. In the proposed approach, multiscan nodes rely on using their (potentially idle) second wireless interface to opportunistically scan and preassociate with alternative Access Points (APs) and eventually seamlessly handover ongoing connections. A novel handover scheme based on on-vehicle antennas is introduced in [8], and the numerical analysis results show that the proposed scheme can pre-trigger handover appropriately, which ensures a higher handover success rate and has an improvement on system throughput.

Coordinated multiple point transmission (CoMP) transmission and reception allows geographically separated base stations to joint sending data to one terminal and joint receiving data from one terminal, by which the inter-cell interference could be reduced and the system frequency spectral efficiency would be improved. In [9], CoMP is introduced to solve inter-cell interference issues, and the simulation results show that the CoMP schemes achieve different gains in average sector throughput and 5\% edge-user throughput gain as compared to that of conventional pre-coding scheme. Zhou and Wan [10] proposed an approach to improve the throughput of systems applied CoMP by adjust Time advance (TA). A new handover scheme was designed for CoMP scenarios in [11], and the handover model of CoMP system was analyzed and the signaling transmission procedure of CoMP cooperating sets handover was designed. Unfortunately the system performance was not discussed.

From the analysis above we can see that, there are few researches on the application of base stations interaction and multiple vehicle stations cooperation. In order to take full advantage of the multiple base stations cooperation feature of CoMP systems under highspeed scenarios, this article proposes a seamless soft handover scheme based on CoMP, which allows the train to receive signals from both adjacent base stations when the train travels through the overlapping areas. Thus, the handover failure rate is degraded and the reliability of train to ground communication is guaranteed.

The rest parts of the article are arranged as follow: Section 2 introduces the current handover scheme in LTE systems. Section 3 describes the proposed handover scheme in detail. Section 4 analyzes the system performance. Section 5 illustrates the simulation results and the improvement achieved by the proposal. And finally Section 6 concludes the whole article.

\section{Conventional hard handover scheme in LTE systems}

The current hard handover scheme in LTE systems is shown in Figure 1 . The eNodeB is with the coverage radius $R$. The width of overlapping area is $L$. The vertical distance between a eNodeB and track is $d_{\text {min }}$. The whole railway mobile communication network has a feature of linear coverage topology. The overall handover procedure could be described as follow.

As the train moves into the overlapping area from cell $i$, eNodeB $i$ decides whether to handover or not according to the reported received signal strength indication (RSSI), reference signal received power (RSRP) or reference signal received quality (RSRQ) measurement information by the train and the radio resource management (RRM) information, as shown in Figure 1a. Once the handover is triggered, the train disconnects with eNo$\mathrm{deB} i$ and tries to synchronize with the target eNodeB $j$. Then the Mobility Management Entity (MME) switches communication route and the previous station eNodeB $i$ releases both user plane resources and control plane resources when the handover procedure is completed, as shown in Figure 1b.

As analyzed above, the current handover scheme in LTE systems is a Break-Before-Make approach. The scheme allows the train to receive signals from only one base station at one time. It will cause a larger outage

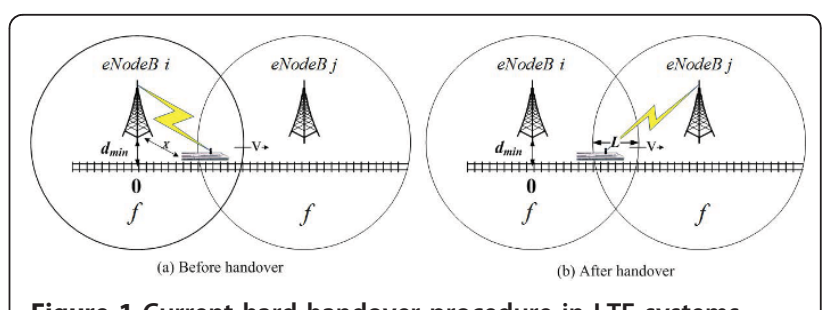

Figure 1 Current hard handover procedure in LTE systems. 
probability and interrupt latency, which severely affect the reliability of train to ground communication.

\section{A CoMP based soft handover scheme in LTE systems}

\subsection{A co-channel deployment approach for railway} communication

In order to achieve CoMP joint processing and transmission between the two adjacent eN-odeBs along the railway track, an interference-avoid co-channel deployment approach is proposed in this article.

As OFDM is used in LTE, the inter-cell interference is the main source of interference. To avoid the possible large inter-cell interference when train travels through the overlapping region, this article proposes a frequency allocation approach for railway scenario: ignoring the reserved dedicated resources, we divided the whole frequency band into two parts which are called F1 and F2 respectively, as shown in Figure 2. By doing so the proposed co-channel network approach is given in Figure 3: $\mathrm{F} 1$ is assigned to the up-direction trains (the trains are going to the metropolis) and F2 is assigned to the down-direction trains (contrary to up-direction). Compared with the existing GSM-R network whose typical frequency reuse factor is 3 , the proposed co-channel deployment approach can not only maximize the spectrum efficiency but also enable the interactions of adjacent eNodeBs.

\subsection{Dual on-vehicle stations collaborative scheme}

For reliability and throughput, the transmission latency is detrimental. In order to eliminate the transmission delay, this article proposes a dual on-vehicle stations cooperation scheme, which takes full advantage of the distributed antennas transmission and the body length

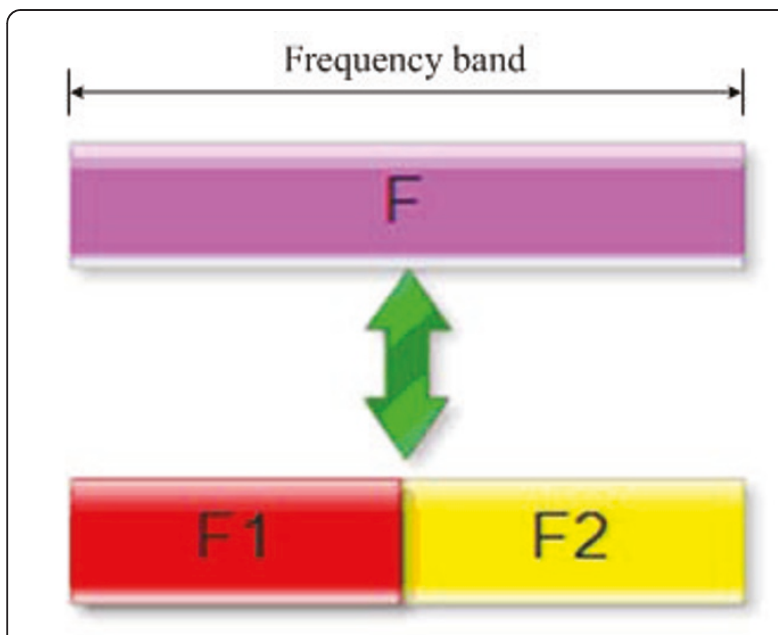

Figure 2 The frequency allocation approach.

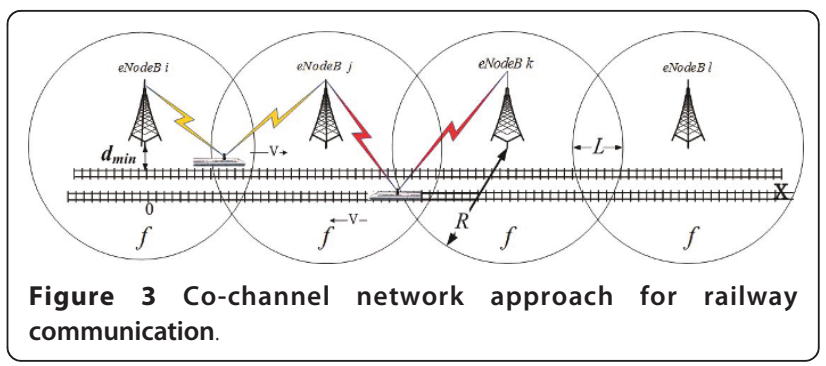

of high speed train. The mobile relay stations (MRSs) controlled by central control station (CCS) are mounted in the front and the rear of the train. Figure 4 shows the schematic diagram of the scheme. Antennas 1 and 2 belong to the front station and the rear station respectively. The uplink data of the users inside the train are gathered to the CCS by pico-base stations deployed onvehicle, and then the front and the rear stations transmit the gathered data to eNodeBs along the track under the control of CCS. Meanwhile, the downlink data received by the two on-vehicle stations form eNodeBs along the track are gathered to the CCS, and then the CCS forwards the collected data to the pico-base stations inside the train. With the above procedure having been done, the communication between users and eNodeBs along the track can be successfully achieved.

This scheme can solve the 'processing capacity bottleneck' problem caused by the conventional single onvehicle station scheme. Moreover, a good diversity gain would be obtained since the distance between Antennas 1 and 2 is far away enough.

\subsection{CoMP based soft handover scheme}

This article proposes a seamless soft handover scheme utilizing CoMP joint processing and transmission technology, which can significantly improve the handover performance when the train moves through the overlapping areas.

As shown in Figure 5, as the front on-vehicle station enters into the overlapping area, the source eNodeB $i$ activates the cooperative transmission set (CTS) composed of eNodeB $i$ and eNodeB $j$. The two or more eNodeBs communicating with MRSs simultaneously are called CTS. The CTS activation is based on the measurement information reported by the moving train and the position information supplied by the communication

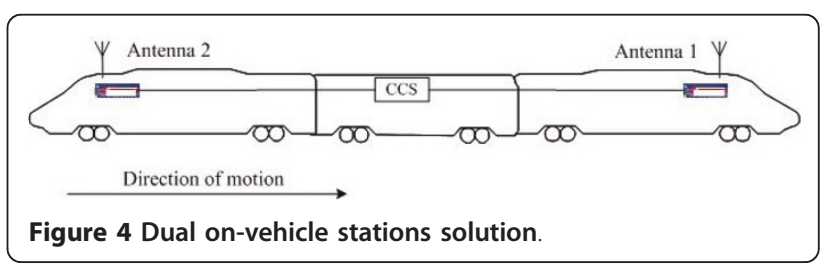




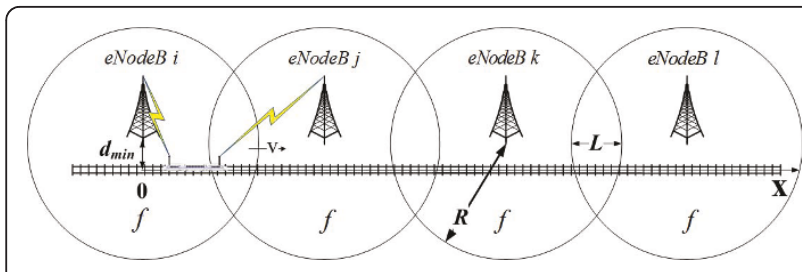

Figure 5 The target eNodeB joins the cooperative set.

based train control system (CBTC). Once the CTS is activated, the source eNodeB $i$ shares all the user plane data of users inside the train to the target eNodeB $j$ by the high-speed backhaul of LTE network. The two adjacent eNodeBs both use the same frequency resource to communicate with the train. Signals from the eNodeBs in the cooperative set are in-phase superposed by precoding, which provides a diversity gain and power gain. It should be noted that CoMP CTS always contains two eNodeBs in the linear coverage topology of high-speed railway.

As shown in Figure 6, eNodeB $i$ and eNodeB $j$ keep the cooperative relation and communicate with the train simultaneously when the train body entirely enters into the overlapping area. Both the front and the rear onvehicle stations can receive signals from the two cooperative eNodeBs, but the measurement information is only forwarded to the source eNodeB $i$. The source eNodeB $i$ decides whether to handover or not according to the measurement information reported by the moving train and the RRM information of the target eNodeB $j$, without interrupting the data transmission in user plane.

The signal from the source eNodeB $i$ drops gradually as the train moves farther away from it. Once the signal strength of target eNodeB $j$ is larger than that of source eNodeB $i$ for $h \mathrm{~dB}$, the handover is triggered. The signaling switch in control plane is a 'break before make' process, which is similar to current hard handover scheme. Meanwhile, both eNodeB $i$ and eNodeB $j$ transmit the user plane data to users inside the train with the same frequency resource by Physical Downlink Shared Channel (PDSCH) without interrupting communications, which avoids the interrupt latency caused by current hard handover in LTE systems. After the handover

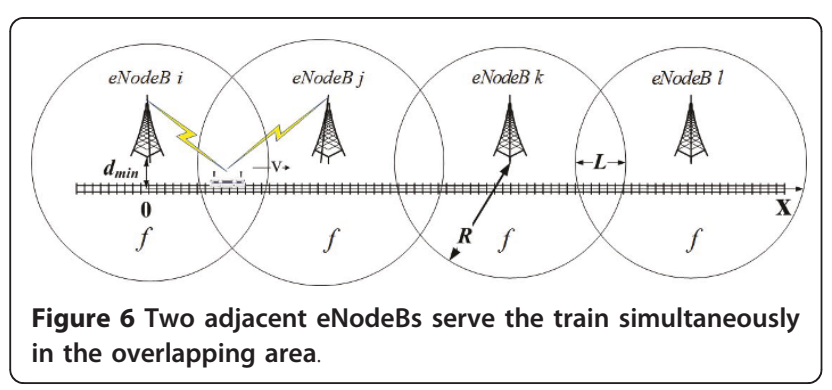

procedure having been done, the new source eNodeB $j$ kicks the former source eNodeB $i$ off the cooperative set, when the signal strength of eNodeB $i$ drops to a certain threshold, as shown in Figure 7.

Summarily, the optimized handover scheme based on CoMP can significantly degrade the outage probability and improve the handover performance, by which the train can communicate with the two adjacent eNodeBs in the overlapping area. The scheme achieves a seamless handover performance as soft handover.

\section{Performance analysis}

In high-speed environments, the Doppler Effects would lead to irreducible bit error rate (BER) which is called error floor $[12,13]$. However, according to technical specifications (TS) of LTE $[14,15]$, the procedure of triggering handover contains three phases: the user equipments (UEs) measure the RSSI, RSRP or RSRQ, sent the measurement reports to source eNodeB, and then the radio resource control (RRC) of source eNodeB decides whether handover is triggered or not [16]. The 3GPP evaluation documents [17] also point out that the handover measurement and radio link failure (RLF) only depend on the RSSI, RSRP or RSRQ. Though the BER performance would degrade the QoS, if the RSRP remains above a certain threshold for a fixed duration, the wireless link will be re-established and assured to complete the handover. At most of time, the high-speed train travels through the wide plain and viaduct, the line-of-sight (LOS) path experienced free-space loss only between MRS and BSs is available and there are few reflectors or scatterers. The major influence on wireless channel caused by relative motion between transmitter and receiver is Doppler shift instead of Doppler spread. Therefore in high-speed railway scenario, instead of considering Doppler Effects which degrades BER, we only need to consider Doppler shift which would impair handover performance. In [18], Doppler shift in the overlapping region of two neighboring eNodeBs (handover region) is almost unchanged, and can be compensated [19]. In this article we suppose that Train Control Information, such as train's velocity and location, can be shared by the ground eNodeBs, and Doppler shift has been compensated $[20,21]$.

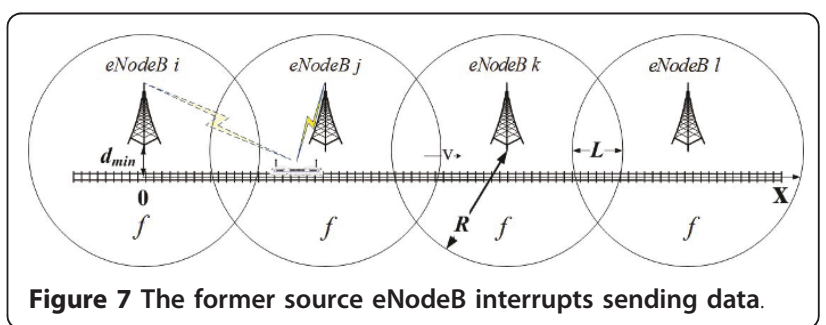


Without loss of generality, let the train be located at $x$ away from the eNodeB $i$, the received power in $\mathrm{dBm}$ in decibels can be expressed as

$$
R(i, x)=P_{t}-P L(i, x)-A(i, x, \sigma)
$$

where $P_{t}$ is the transmit power in $\mathrm{dBm}, P L(i, x)$ is the path loss between eNodeB $i$ and location $x$, and $A(i, x, \sigma)$ is the shadow component at the location $x$, generally modeled as a Gaussian random variable with mean zero and standard deviation $\sigma$. Typically, $\sigma$ is 6 or 8dBin [22].

As the railway communication network is the linear coverage topology, we consider a two-cell system. The shadow fading losses of the two adjacent eNodeBs are correlated and can be expressed as follow according to [23].

$$
A_{i}=a \xi_{0}+b \xi_{i}
$$

where $a^{2}+b^{2}=1$. Note that $i$ is the link number. $\xi_{0}$ is common to both $A_{1}$ and $A_{2}, \xi_{i}$ represents the independent part between the two adjacent eNodeBs. Furthermore, both $\xi_{0}$ and $\xi_{i}$ are independent Gaussian random variables with mean zero and standard deviation $\sigma$. Meanwhile, because the tail of Gaussian distribution extends to infinity, a fade margin $F \mathrm{~dB}$ is added to the transmit power.

\subsection{Outage in the CoMP handover scheme}

In our proposal, the two adjacent eNodeBs communicate with the train simultaneously in the overlapping area. Outage will happen if and only if both signals are of unacceptable quality. So the outage probability using the proposed handover scheme is

$$
P_{\text {CoMP_handover }}=\operatorname{Pr}[\min (A(i, x, \sigma), A(j, x, \sigma))>F]
$$

where, $A(i, x, \sigma)$ and $A(j, x, \sigma)$ represent the shadow fading losses of eNodeB $i$ and eNodeB $j$ respectively. $F$ is the fade margin and $F=P_{t}-P L-R_{s} . P L$ is the path loss and $R_{s}$ is the receiver sensitivity. By straightforward manipulation, the outage probability can be shown as

$$
\begin{aligned}
& P_{\text {Comp_handover }}=\frac{1}{(2 \pi \sigma)^{3 / 2}} \int_{-\infty}^{\infty} e^{-\frac{t^{2}}{2 \sigma^{2}}} \\
& \left(\int_{\frac{F-a t}{b}}^{\infty} e^{-\frac{\xi_{i}^{2}}{2 \sigma^{2}} d \xi_{i}} \int_{\frac{F-a t}{b}}^{\infty} e^{-\frac{\xi_{j}^{2}}{2 \sigma^{2}} d \xi_{j}}\right) d t \\
& =\frac{1}{\sqrt{2 \pi}} \int_{-\infty}^{\infty} e^{-\frac{x^{2}}{2}}\left[\Phi\left(\frac{F-a \sigma x}{b \sigma}\right)\right]^{2} d x \\
& =\frac{1}{\sqrt{2 \pi}} \int_{-\infty}^{\infty} e^{-\frac{x^{2}}{2}}\left[\frac{1}{2} \operatorname{erfc}\left(\frac{F-a \sigma x}{\sqrt{2} b \sigma}\right)\right]^{2} d x
\end{aligned}
$$

where

$$
\Phi(x)=\int_{x}^{\infty} \frac{1}{\sqrt{2 \pi}} e^{-\frac{t^{2}}{2}} d t, \operatorname{erfc}(x)=\frac{2}{\sqrt{\pi}} \int_{x}^{\infty} e^{-t^{2}} d t
$$

\subsection{Outage in current hard handover scheme}

In the current handover scheme, the MRS can connect to only one eNodeB at one time. For ideal case, the MRS is always switched to the eNodeB with the best signal quality. However, this may lead to the well-known 'pingpong' effect around the cell boundary. In practical systems, handover will be triggered on the condition that the received power of the source eNodeB is lower than that of the target eNodeB by hysteresis level $h$.

In most of the existing researches, the outage probability in hard handover system is obtained with the hysteresis level $h$ being assumed infinite so that it is impossible to handover to the neighboring cell. This scenario is called an isolated cell. Let shadow fading loss $A=\xi$, where $\xi$ is Gaussian random variable with mean zero and standard deviation $\sigma$. An outage occurs when the fading component is larger than the fade margin, which is expressed as

$$
\begin{aligned}
P_{\text {isolated }} & =\operatorname{Pr}(\xi>F) \\
& =\frac{1}{\sqrt{2 \pi} \sigma} \int_{F}^{\infty} e^{-\frac{\xi^{2}}{2 \sigma^{2}}} d \xi \\
& =\Phi\left(\frac{F}{\sigma}\right)=\frac{1}{2} \operatorname{erfc}\left(\frac{F}{\sqrt{2} \sigma}\right)
\end{aligned}
$$

It is well known that the hysteresis is finite in practical scenarios and varied according to different communication scenario. In order to make a fair comparison between conventional scheme and our proposal, a finite hysteresis level is assumed in this article. A handover occurs when the received power of the target eNodeB $j$ is larger than that of the source eNodeB $i$ by $h \mathrm{~dB}$, so the handover probability can be expressed as

$$
P_{x}(i, j)=\operatorname{Pr}[R(j, x)-R(i, x) \geq h]
$$

When the train is located exactly at the midpoint of overlapping region, the outage probability is composed of three parts:

(1) If both signals from the two adjacent eNodeBs can't be received by the train, outage will happen. In such case, the outage probability $P_{\text {both_outage }}$ can be expressed as

$$
P_{\text {both_outage }}=\operatorname{Pr}[A(i, x, \sigma)>F \& A(j, x, \sigma)>h]
$$


(2) Before handover, if the signal strength of source eNodeB is unacceptable and that of target eNodeB is not large enough to trigger handover, this is obviously an outage. Let $\varepsilon_{i}$ be the event that the train is connecting to eNodeB $i$, and $h$ be the hysteresis level. The outage probability $P_{\text {before_ HO can be }}$ expressed as

$$
\begin{aligned}
P_{\text {before_HO }}= & \operatorname{Pr}\left[\varepsilon_{i} ; A(j, x, \sigma)<F<A(i, x, \sigma)\right] \\
= & \operatorname{Pr}[A(i, x, \sigma)-h<A(j, x, \sigma)<F<A(i, x, \sigma)] . \\
& \operatorname{Pr}\left[\varepsilon_{i} \mid A(i, x, \sigma)-h<A(j, x, \sigma)<F<A(i, x, \sigma)\right]
\end{aligned}
$$

where

$$
\begin{aligned}
\operatorname{Pr}[A(i, x, \sigma)-h<A(j, x, \sigma)<F<A(i, x, \sigma)] \\
=\frac{b^{2}}{(2 \pi)^{3 / 2}} \int_{-\infty}^{\infty} e^{-\frac{t^{2}}{2}} \\
\frac{F+h-a \sigma t}{\int_{\frac{F-a \sigma t}{b \sigma}}} \frac{F-a \sigma t}{b \sigma} \int_{x_{2}-\frac{h}{b \sigma}}^{-\frac{\left(x_{1}-a t\right)^{2}+\left(x_{2}-a t\right)^{2}}{2 b^{2}}} d x_{1} d x_{2} d t
\end{aligned}
$$

In fact, the correlation of shadow fading losses would decrease with the increasing angle-of-arrival difference [24]. We assume that there is no site-to-site correlation (i.e, $a=0$ ) as the angle-of-arrival difference is quite large. Thus, the above expression can be simplified as

$$
\frac{1}{2 \pi} \int_{\frac{F}{\sigma}}^{\frac{F+h}{\sigma}} \int_{x_{2}-\frac{F}{\sigma}}^{\frac{F}{\sigma}} e^{-\frac{x_{1}^{2}+x_{2}^{2}}{2}} d x_{1} d x_{2}
$$

(3) After handover, although the train has been successfully switched to the target eNodeB, outage will happen if the signal strength of the target eNodeB is too weak. It should be noted that the target eNodeB is determined by the direction to which the train moves towards, that is, the train can't be switched to the previous source eNodeB though the signal strength of the novel source eNodeB is terrible. Thus, the outage probability $P_{\text {after }}$ HO can be expressed as

$$
\begin{aligned}
P_{\text {after_HO }}= & \operatorname{Pr}\left[\varepsilon_{j} ; A(i, x, \sigma)<F<A(j, x, \sigma)\right] \\
= & \operatorname{Pr}[A(i, x, \sigma)<F<A(j, x, \sigma) \\
& \cdot \operatorname{Pr}\left[\varepsilon_{j} \mid A(i, x, \sigma)<F<A(j, x, \sigma)\right]
\end{aligned}
$$

where

$$
\begin{aligned}
& \operatorname{Pr}[A(i, x, \sigma)<F<A(j, x, \sigma)]= \operatorname{Pr}\left[a \xi_{0}+b \xi_{i}<F<a \xi_{0}+b \xi_{i}\right] \\
&= P\left(\frac{\xi_{0}}{\sigma}=t\right) \cdot P\left(\frac{\xi_{i}}{\sigma}<\frac{F-a \xi_{0}}{b \sigma}\right) \\
& \cdot P\left(\frac{\xi_{j}}{\sigma}>\frac{F-a \xi_{0}}{b \sigma}\right) \\
&= \frac{1}{(\pi)^{3 / 2}} \int_{-\infty}^{\infty} e^{-\frac{t^{2}}{2}} \\
& \int^{\infty} \frac{F-a \sigma t}{b \sigma} \int_{0}^{-\frac{x_{1}^{2}+x_{2}^{2}}{2}} d x_{1} d x_{2} d t
\end{aligned}
$$

It should be noted that the value of second item in (9) is difficult to obtain analytically. Whether the train is connecting to eNodeB $i$ or not can not be determined by a snapshot of the system. It is generally assumed to be $1 / 2$ in the midpoint of the overlapping region. Since $A_{j}<A_{i}$ in (9), that is, the attenuation loss to eNodeB $j$ is smaller, and the train has a higher chance to connect to eNodeB $j$. Following [24], this probability is chosen as 0.6 .

From the analysis above, the overall outage probability using hard handover scheme can be expressed as

$$
P_{\text {hard_outage }}=P_{\text {both_outage }}+P_{\text {before_HO }}+P_{\text {after_HO }}
$$

\section{Simulation and analysis}

To make a comparison between current scheme and our proposal, the MATLAB simulation results and analysis are presented. Detailed simulation parameters are shown in Table 1 [22] As the train moves through the overlapping area, the received signal strength of the two adjacent eNodeBs is shown in Figure 8. The red and green curves represent the received signal strength of eNodeB $i$ and that of eNodeB $j$ respectively. Due to the influence of path loss and shadow fading loss, both signals

Table 1 Simulation parameters [22]

\begin{tabular}{ll}
\hline Parameters & Value \\
\hline Channel bandwidth (BW) & $10 \mathrm{MHz}$ \\
Antenna pattern & Omni \\
Carrier frequency & $2.5 \mathrm{Ghz}$ \\
$P_{t}$ & $46 \mathrm{dBm}$ \\
Height of antenna: $h_{\text {at }}$ & $1.5 \mathrm{~m}$ \\
Height of eNodeB: $h_{\mathrm{eNodeB}}$ & $35 \mathrm{~m}$ \\
$d_{\text {min }}$ & $30 \mathrm{~m}$ \\
$N_{0}$ & $-174 \mathrm{dBm} / \mathrm{Hz}$ \\
Cell radius $(R)$ & $3 \mathrm{~km}$ \\
Site-to-site distance & $4.8 \mathrm{~km}$ \\
Path loss model & ITU-R M.1225 \\
\hline
\end{tabular}




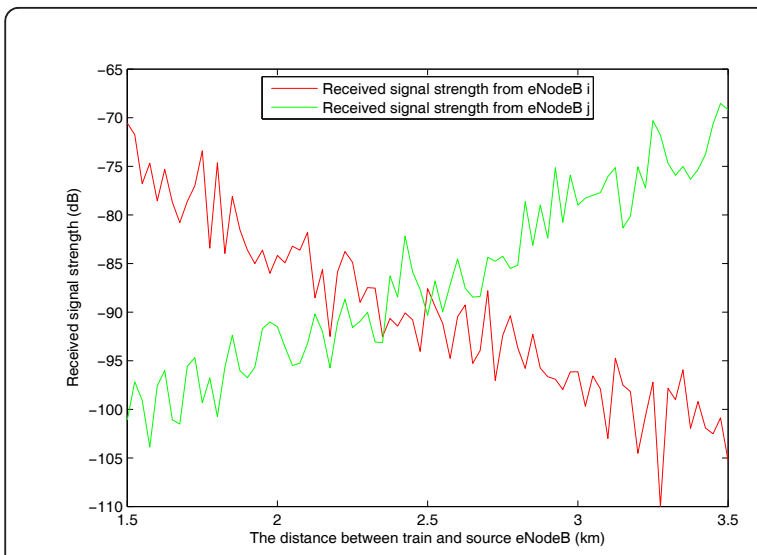

Figure 8 Received signal strength according to train location.

strength of eNodeB $i$ and eNodeB $j$ are extremely lower at cell boundary than those in the interior of a cell, which would cause a higher handover failure probability.

The outage comparison between the current handover scheme in LTE systems and our proposal when $\sigma=6$ and $h=6 \mathrm{~dB}$ is shown in Figure 9. From the figure, it can be seen that our proposed hard handover estimation provides a rather tight bound, compared with existing isolated cell estimation. Meanwhile, the CoMP soft handover scheme provides a significant improvement in outage probability compared with that in current hard handover scheme systems, which can be easily obtained from the figure.

Compared with Figures 9, 10 and 11, we can see the outage probability comparison with different hysteresis level and standard deviation of shadow fading loss.

Additionally, from these figures, with the increasing of hysteresis level and standard deviation of shadow fading loss, both the current handover scheme in LTE systems and our proposal experience a higher outage probability.

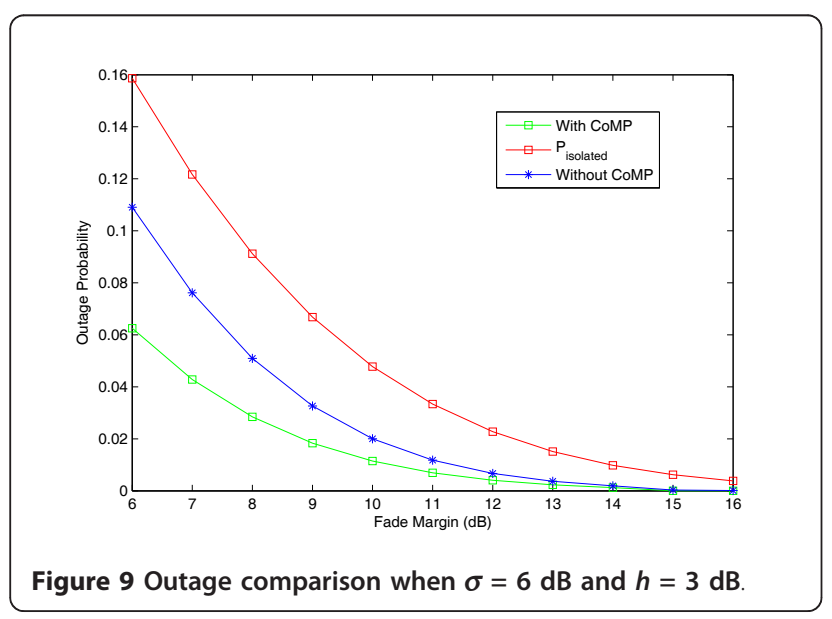

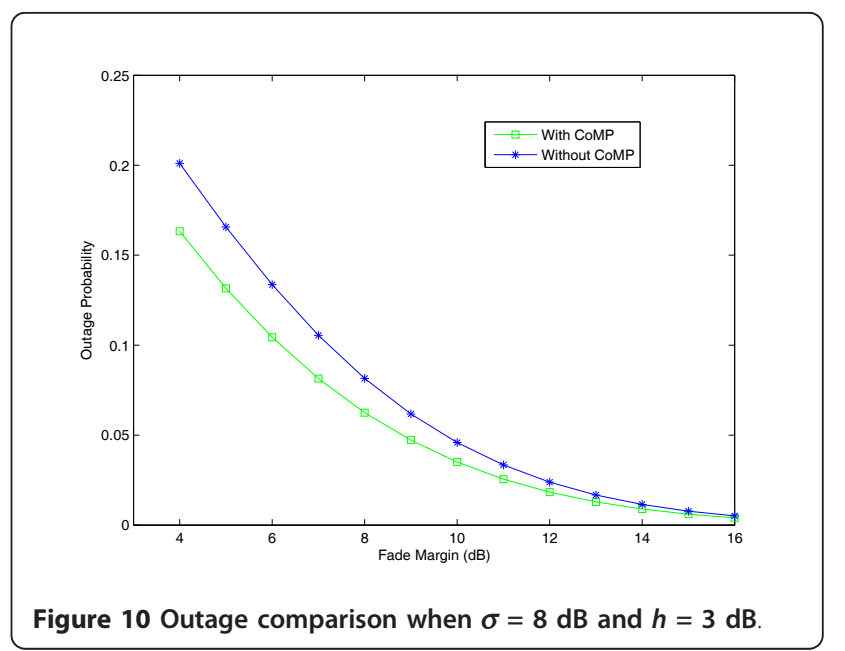

However, the proposals provide the better performance as the hysteresis increases.

In Figure 12, the outage probability using different schemes is given according to distance. The green and the blue curves represent outage probability using current hard handover scheme in LTE systems connecting to source eNodeB and target eNodeB, respectively. The red curve shows outage probability using the proposal. From the figure, it could be seen that the proposal provides a quite lower outage probability, especially in the midpoint of the overlapping area, where the received signal strength from both eNodeBs is pretty low.

In addition, Figure 13 provides a comparison of handover success probability according to the location of the train. The red and green curves denote the handover success probability of hard handover scheme and that of the proposal respectively. From the figure, it can be clearly seen that the proposal can significantly improve the handover performance with the successful handover probability increasing from 89 to around $99 \%$.

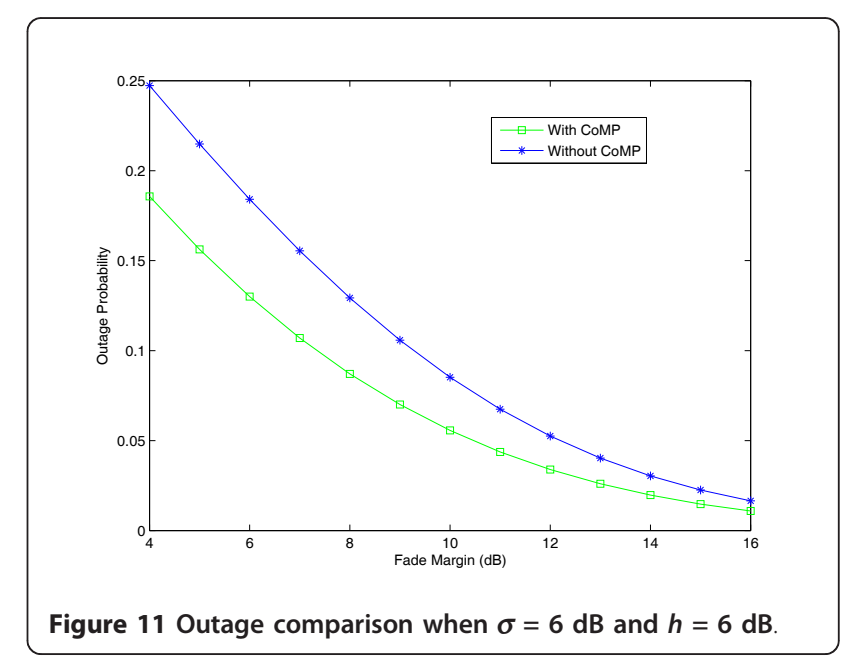




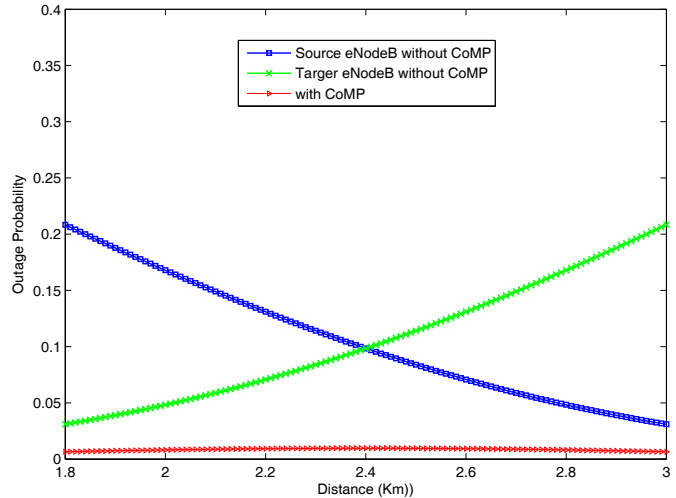

Figure 12 Outage probability according to distance

\section{Conclusion}

As the evolution of railway mobile communication system is confirmed by UIC, LTE technology becomes a potential solution for future railway systems. The railway communication system has stringent requirements for wireless communication availability and latency. However the frequent handover caused by high-speed movement will seriously affect the performance. This article proposes a CoMP based soft handover scheme for LTE system in high speed railway, meanwhile, a co-channel deployment approach for railway communication and dual on-vehicle station coordination mechanism is proposed too. The proposal allows the train to receive signals of both adjacent eNodeBs, which significantly improves the handover performance and degrades the outage probability, as the simulation results show. It is worth mentioning that signaling interactions in control plane and performance analysis on MAC layer are the focuses in our following work.

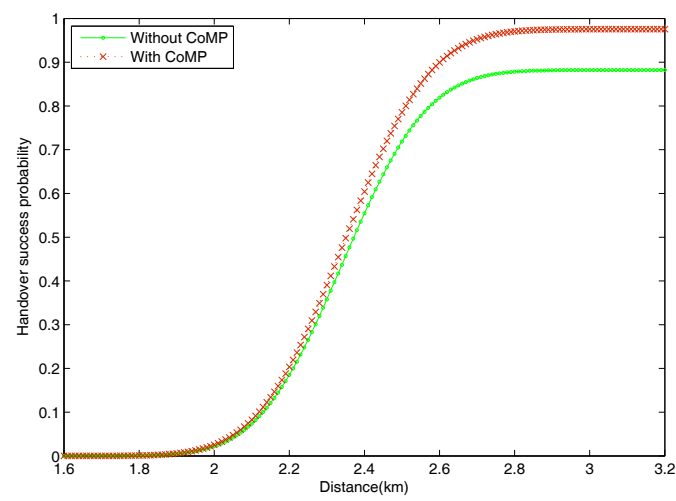

Figure 13 Comparison of handover success probability of different schemes.

\section{Acknowledgements}

The work of the authors' was supported partially by the 973 Program under the Grant 2012CB316100, NSFC under the Grant 61071108, 61032002, the Key Program of Technological R\&D of the Ministry of Railway under the Grant 2011X011-A, and the ZTE R\&D Foundation.

\section{Author details}

${ }^{1}$ Institute of Mobile Communications, Southwest Jiaotong University, Chengdu, 610031, China ${ }^{2}$ College of Physics and Electronic Engineering, Guangxi University for Nationalities, Nanning, 530006, China

\section{Competing interests}

The authors declare that they have no competing interests.

Received: 6 February 2012 Accepted: 13 June 2012

Published: 13 June 2012

\section{References}

1. H Jijing, M Jun, ZH Zhangdui, Research on handover of GSM-R network under high-speed scenarios. Railway Commun Signals. 42, 51-53 (2006)

2. K Tsagkaris, ND Tselikas, N Pleros, A handover scheme based on moving extended cells for $60 \mathrm{GHz}$ radio-over-fiber networks, in Proceedings of IEEE International Conference on Communications, Dresden, Germany, 1-5 (14-18 June 2009)

3. N Pleros, K Vyrsokinos, K Tsagkaris, ND Tselikas, A $60 \mathrm{GHz}$ radio-over-fiber network architecture for seamless communication with high mobility. Lightwave Technol. 27, 1957-1967 (2009)

4. S Jeon, S Lee, A relay-assisted handover technique with network coding over multihop cellular networks. IEEE Commun Lett. 11, 252-254 (2007)

5. R Pabst, BH Walke, DC Schultz, Relay-based deployment concepts for wireless and mobile broadband radio. IEEE Commun Mag. 42, 80-89 (2004)

6. P Bahl, A Adya, J Padhye, A Walman, Reconsidering wireless systems with Multiple radios. Comput Commun Rev. 34, 1-8 (2004)

7. V Brik, A Mishra, S Banerjee, Eliminating handoff latencies in 802.11 WLANs using multiple radios: applications, experience, and evaluation, in Proceedings of the 5th ACM SIGCOMM conference on Internet Measurement, New York, USA, 22-23 (2005)

8. C Yang, L Lu, C Di, X Fang, An on-vehicle dual-antenna handover scheme for high-speed railway distributed antenna system, in Proceedings of the 6th IEEE International Conference on Wireless Communicaitons Networking and Mobile Computing, Chengdu, China, 1-5 (Sept 2010)

9. Z Wang, Y Wang, C Lin, Q Wang, Enhanced downlink MU-CoMP schemes for TD-LTE-advanced, in Proceedings of IEEE Wireless Communications and Networking Conference, Sydney, Australia, 1-6 (18-21 Apr 2010)

10. M Zhou, L Wan, Anaysis into timing advance issue in CoMP systems, in Proceedings of the 70th IEEE International Conference on Vehicular Technology, Anchorage, USA, 1-5 (20-23 Sept 2009)

11. $X X u, X$ Chen, J Li, Handover scheme for coordinated multi-point transmission/reception system. ZTE Commun. 1, 32-36 (2010)

12. H Fu, PY Kam, Simple error probability derivation for binary DPSK over fast Rician channels with diversity. IEEE Electron Lett. 42, 163-165 (2006). doi:10.1049/el:20063414

13. H Fu, PY Kam, Effect of Doppler shift on performance of binary DPSK over fast Rician fading channels with diversity reception, in Proceedings of IEEE International Symposium on Information Theory and Its Applications, Toronto, Canada, 1-6 (7-10 Dec 2008)

14. 3GPP TS 36.331 V9.4.0, Radio Resource Control (RRC), http://www.3gpp.org/ $\mathrm{ftp} / \mathrm{Specs} /$ archive/36 series/36.331/

15. 3GPP TS 36.300 V10.0.0, E-UTRA and E-UTRAN Overall Description http:// www.3gpp.org/ftp/Specs/archive/36 series/36.300/

16. S Sesia, LTE - UMTS Long Term Evolution: From Theory to Practice, (Wiley, Chichester, 2009)

17. 3GPP TSG-RAN WG1 \#56bis, R1-091578, Evaluation model for Rel-8 mobility performance, http://www.3gpp.org/ftp/tsg ran/WG1 RL1/TSGR1 56b/Docs/

18. 3GPP TS 36.101 V10.0.0, User Equipment (UE) radio transmission and reception http://www.3gpp.org/ftp/Specs/archive/36 series/36.101/

19. M Morelli, C-CJ Kuo, M-O Pun, Synchronization techniques for orthogonal frequency division multiple access(OFDMA), a tutorial review. Proc IEEE. 95, 1394-1427 (2007)

20. Olli Piirainen, U.S. Patent 6,473,594 (Oct 2002) 
21. Ralf Klotsche, Klaus Wunstel, Thomas-Rolf Banniza, U.S. Patent 7,653,347 (Jan 2010)

22. Guidelines for evaluation of radio transmission technologies for IMT-2000 http://www.itu.int/rec/R-REC-M.1225-0-199702-I/en

23. AJ Viterbi, AM Viterbi, KS Gilhousen, E Zehavi, Soft handoff extends CDMA cell coverage and increases reverse link capacity. Sel Areas Commun. 12, 1281-1288 (1994). doi:10.1109/49.329346

24. Fading Models, http://www.comlab.hut.fi/opetus/333/2004 2005 slides/ Fading models.pdf

doi:10.1186/1687-1499-2012-196

Cite this article as: Luo et al: A CoMP soft handover scheme for LTE systems in high speed railway. EURASIP Journal on Wireless

Communications and Networking 2012 2012:196.

\section{Submit your manuscript to a SpringerOpen ${ }^{\mathcal{O}}$} journal and benefit from:

- Convenient online submission

- Rigorous peer review

- Immediate publication on acceptance

- Open access: articles freely available online

- High visibility within the field

- Retaining the copyright to your article

Submit your next manuscript at $\gg$ springeropen.com 